\title{
Using the PROMETHEE Multi-Criteria Decision Making Method to Define New Exploration Strategies for Rescue Robots
}

\author{
Patrick Taillandier \\ IRIT - Université Toulouse 1 Capitole \\ 2, rue du Doyen Gabriel Marty \\ Toulouse, France \\ patrick.taillandier@gmail.com
}

\author{
Serge Stinckwich \\ UMMISCO, UMI 209 \\ IRD/IFI/Vietnam National University \\ CNRS, UMR 6072 GREYC \\ F-14032 Caen, France \\ serge.stinckwich@ird.fr
}

\begin{abstract}
The exploration of an unknown environment by a robot system (an individual robot or a team of robots) is a well-studied problem in robotics. This problem has many applications and, among them, the post-disaster search of victims in an urban space. Most of proposed exploration algorithms are based on the use of specific criteria to define the quality of the possible movements. In this paper, we propose an exploration approach based on the combination of several criteria thanks to the PROMETHEE II multi-criteria decision making method. The PROMETHEE II method allows one to establish a complete ranking between possible movements based on outranking relations. Experimental results show that this approach can be used to effectively combine different criteria and outperforms several classic exploration strategies.
\end{abstract}

Keywords: search and rescue robotics, exploration strategies, multiple criteria decision making, PROMETHEE methods

\section{INTRODUCTION}

Improvement in robotics and in artificial intelligence has led to imagining new applications for robots. One of them is the use of robots for search and rescue operations during disasters. Actually, in urban areas, most of the victims die during the first 48 hours partly because they are not detected in time. Moreover, the rescuers themselves face great dangers when exploring the impacted sites.

The mapping task consists in constructing a spatial representation of an unknown environment by robotic means. This map is particularly useful during search and rescue operations over a disaster area, where we want to search for points of interest or victims. To construct this map, the robots must move through the environment to acquire data. An exploration strategy determines the future positions of robots when they explore the environment. The aim of the exploration process is to cover the whole environment in a minimum period of time.

These algorithms are based on different criteria allowing the robots to make the pertinent decisions concerning their next displacement step. In this paper, we propose an exploration approach based on the combination of these criteria. Our approach use the PROMETHEE II multi-criteria decision making method in order to select the best decision the robots have to make according to the context. In particular, our approach allows to simply add context-specific criteria to improve the exploration efficiency. In Section 2, the general context of our work is introduced. Section 3 is devoted to the presentation of the multi-criteria decision approach we propose to use. Section 4 present some experiments carried out. Section 5 concludes and presents the perspectives of this work.

\section{CONTEXT OF THE WORK}

The definition of strategies for autonomous exploration of unknown environments has been addressed by several works in literature. Most of them are based on the Next-Best-View (NBV) schema. In this family of algorithms, the exploration is viewed as a sequence of two steps: a motion toward a location and a new observation of the environment done by the robot at this location. The location is chosen among a set of candidate locations located on the frontier between free known space and unexplored part of the environment. The first one to introduce this approach was Yamauchi with his seminal work on frontierbased exploration in 1998[1].

\section{A. Criteria for NBV exploration strategies}

In order to evaluate candidate locations $p$, a variety of criteria for NBV-related exploration strategies has already been proposed previously in literature:

- No criteria: the next location for observation is chosen in a random way. This strategy might be useful as a bottom line for comparison with other strategies.

- $L(p)$ [1]: length of the minimum collision-free path to $p$.

- $A(p)$ [2]: new information acquired from $p$ and obtained by simulating a perception from $p$.

- $O(p)$ [3]: overlap between the current map and the area visible from $p$. It evaluates the ease of robot localization and is calculated as the length of the obstacle line segments which are visible from $p$.

- $P(p)$ [4]: probability that the robot will be able from $p$ to transmit information to the base station. 


\section{B. Combine Criteria}

Some approaches use several of these criteria to combine them in an ad hoc way in order to obtain more complex utility functions. Most of the time, the result cannot be theoretically justified (except for the ACG strategy). The criteria used are not always of the same nature which makes them not easily combinable. It is difficult in these conditions to add new criteria.

- $A(p)$ and $L(p)$ are linearly combined in [5]:

$$
u(p)=A(p)-\beta L(p)
$$

- Gonzáles-Baños-Latombe (GBL) strategy[2] used a evaluation fonction defined on a candidate $p$ as:

$$
u(p)=A(p) * \exp (-\lambda L(p))
$$

where $\lambda$ is a positive constant value. $\lambda$ is a weight that can be modified in order to adjust the cost of $A$ versus $L$. This formula is validated with experimental setup but he is not well justified from a theoretical point of view.

- Amigoni-Caglioti-Galtarossa (ACG) strategy[6] is based on the concept of relative entropy presented by the same authors and theoretically founded in information theory. This approach mixes the expected information of the sensing activity and the distance travelled by the robot.

- Burgard strategy[7] is a probabilistic approach for multirobot exploration. This one used also the cost of moving the robot to a location and the utility expected. The optimal path from the current position to all frontier locations used a variant of the "value iteration" markov-decision process algorithm. This non-deterministic approach tends to favor candidate locations in open spaces rather than narrow passages or obstacles.

Most approaches, however, do not consider the problem of searching for interesting features (for example victims) inside the environment, while doing the exploration.

More recently, researchers have proposed to combine criteria with the help of MCDM (Multiple Criteria Decision Making) framework, like the Choquet fuzzy integral [3], [4]. The main advantage of this approach is the possibility to take into account relations between criteria like redundancy or synergy explicitively.

\section{APPROACH PROPOSED}

As introduced in Section II. numerous strategies were defined in literature for the robot exploration problem. In this paper, we propose to follow the same general approach as the one proposed by Basilico and Amigoni [3],[4] and to use a multi-criteria decision making framework to combine the different criteria. However, instead of using the Choquet fuzzy integral, we propose to use the alternate PROMETHEE II method to make decision.

This section is organized as follows. In section III-A, we first describe state of the art of multi-criteria decision making methods. Section III-B is dedicated to the presentation of the multi-criteria decision making method that we propose to use, the PROMETHEE II method. Section III-C presents an example of use of the PROMETHEE II method with some well-known criteria. Finally section III-D compare our approach to the one proposed by Basilico and Amigoni [4].

\section{A. State of the art of multi-criteria decision making methods}

In literature, multi-criteria decision making methods are [8] classifies in three groups. The first one, called partial aggregation approaches, consists in comparing the different possible decisions per pair by the means of outranking relations [9], [10]. The second family of approaches, called complete aggregation approaches, consists in aggregating all criteria in a single criterion (utility function) which is then used to make the decision [11], [12], [13]. The last family of approaches, which is highly interactive, consists in devising a preliminary decision and in comparing it with other possible decisions to determine the best one [14], [15].

In this paper, we propose to use the PROMETHEE II method. This method is based on partial aggregation: all possible decisions are compared to each other by pair and ranked. It is then possible to select a priori best decision.

PROMETHEE II method is particularly well-fitted in our case, because it allows to make a decision from incomparable criteria. Thus, it is possible to use criteria that are not directly comparable to characterise a decision: for example, a time criterion and a distance one. Compare to the well-established ELECTRE decision making method (also based on partial aggregation), the PROMETHEE II method is easier to use and allow to get more robust results [16].

\section{B. The PROMETHEE II method}

PROMETHEE II (Preference Ranking Organization Method for Enrichment Evaluations) has been used with success to solve many problems [17]. This method is based on a comparison pair per pair of possible decisions along each criterion. Possible decisions are evaluated according to different criteria, which have to be maximised or minimised. The use of the PROMETHEE II method requires two additional types of information for each criterion: a weight and a preference function.

The preference function characterises the difference for a criterion between the evaluations obtained by two possible decisions into a preference degree ranging from 0 to 1 . In order to ease the definition of these function, six basic preference functions have been proposed in [18].

The decision making process by the PROMETHEE II method is composed of four steps that are detailed hereafter.

Step 1 This step computes for each pair of possible decisions and for each criterion, the value of the preference degree. Let $g_{j}(a)$ be the value of a criterion $j$ for a decision $a$. We note $d_{j}(a, b)$, the difference of value of a criterion $j$ for two decisions $a$ and $b$.

$$
d_{j}(a, b)=g_{j}(a)-g_{j}(b)
$$


$P_{j}(a, b)$ is the value of the preference degree of a criterion $j$ for two decisions $a$ and $b$. The preference functions used to compute these preference degrees are defined such as:

$P_{j}(a, b)=F\left(d_{j}(a, b)\right)$ with $\left.\forall x \in\right]-\infty, \infty[, 0 \leq F(x) \leq 1$

Step 2 This step consists in aggregating the preference degrees of all criteria for each pair of possible decisions. For each pair of possible decisions, we compute a global preference index. Let $C$ be the set of considered criteria and $w_{j}$ the weight associated to the criterion $j$. The global preference index for a pair of possible decision $a$ and $b$ is computed as follows:

$$
\pi(a, b)=\sum_{j \in C} w_{j} \times P_{j}(a, b)
$$

Step 3 The third step, which is the first that concerns the ranking of the possible decisions, consists in computing the outranking flows. For each possible decision $a$, we compute the positive outranking flow $\phi^{+}(a)$ and the negative outranking flow $\phi^{-}(a)$. Let $A$ be the set of possible decisions and $n$ the number of possible decisions. The positive outranking flow of a possible decision $a$ is computed by the following formula:

$$
\phi^{+}(a)=\frac{1}{n-1} \sum_{x \in A} \pi(a, x)
$$

The negative outranking flow of a possible decision $a$ is computed by the following formula:

$$
\phi^{-}(a)=\frac{1}{n-1} \sum_{x \in A} \pi(x, a)
$$

Step 4 The last step consists in using the outranking flows to establish a complete ranking between the possible decisions. The ranking is based on the net outranking flows. These are computed for each possible decision from the positive and negative outranking flows. The net outranking flow $\phi(a)$ of a possible decision $a$ is computed as follows:

$$
\phi(a)=\phi^{+}(a)-\phi^{-}(a)
$$

The higher the value of the net outranking flow for a decision, the better the decision is. In our application context where we are only interested in the best decision to make, we will choose the decision that maximises the net outranking flows.

\section{Example of use of the PROMETHEE II method}

As an illustration on how to apply Promethee II method to the exploration problem, we present examples of preference functions that can be used for the three main criteria defined in [4].

- $L(p)$ : length of the minimum collision-free path to $p$. This preference function (see figure 1) is defined in such a way as small variations of $L$ are not very important to determine if a location is better than another one, whereas big differences are discriminatory. For example, if a location is at $20 \mathrm{~m}$ to the robot and another one at $19.5 \mathrm{~m}$, the difference of value will have a very slight

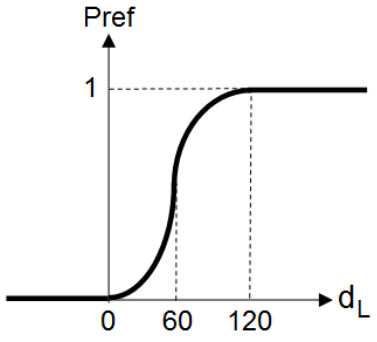

Fig. 1. Preference function for the $L$ criterion. Let $C 1$ and $C 2$ be two candidate locations, $d_{L}(C 1, C 2)=L(C 2)-L(C 1)$

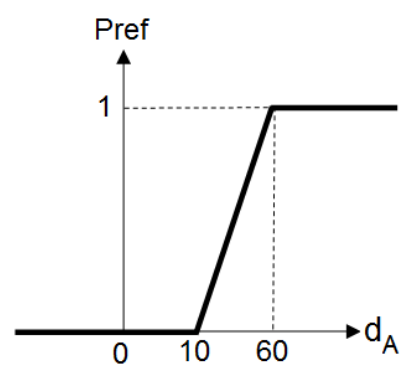

Fig. 2. Preference function for the $A$ criterion. Let $C 1$ and $C 2$ be two candidate locations, $d_{A}(C 1, C 2)=A(C 1)-A(C 2)$

impact on the assertion that the first location is better than the second one. However, if the difference is superior to $10 \mathrm{~m}$, then the closest location has strong chance to be declared better than the second location.

- $A(p)$ : new information acquired from $p$ and obtained by simulating a perception from $p$. Figure 2 presents the preference function defined. This function is defined in such a way that small variations of $A$ has no importance to determine if a location is better than another one. This criterion begins to be accounted only when the difference of value is higher than a given threshold value $\left(10 \mathrm{~m}^{2}\right)$.

- $P(p)$ : probability that the robot, once reached $p$, will be able to transmit information. Figure 3 presents the preference function defined. This function is very similar to the one defined for the previous criterion. It is defined in such a way as small variations of $P$ have no importance to determine if a location is better than another one. This criterion begins to be accounted only when the difference of value is higher than a given threshold value (0.5).

\section{Comparison with the Basilico and Amigoni approach}

We share with Basilico and Amigoni approach [3],[4] the same objective: to propose a general approach allowing to easily and robustly integrate new criteria in the candidate location selection.

However, the methods proposed for the decision making differ: the method we propose is based on partial aggregation (PROMETHEE II) and Basilico and Amigoni approach is based on complete aggregation (Choquet fuzzy integral). In the context of the Basilico and Amigoni approach, the value of a criterion has a direct influence on the general quality of a 


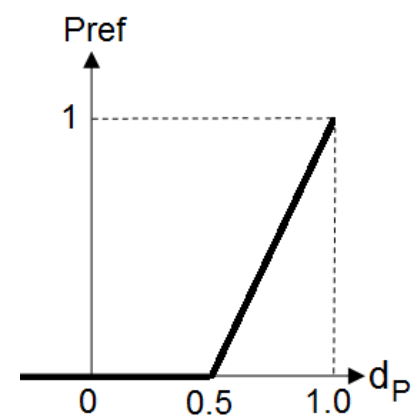

Fig. 3. Preference function for the $P$ criterion. Let $C 1$ and $C 2$ be two candidate locations, $d_{P}(C 1, C 2)=P(C 1)-P(C 2)$

possible location (its utility). In the context of our approach, this influence is indirect: it is the difference of the criterion value between two candidate locations that has a direct impact on the final decision.

The advantage of our approach is to take into account the fact that for some criteria the impact of the criterion difference value is non-linear. Section III-C gives examples of criteria of this kind.

One of the main advantages of the Basilico and Amigoni approach is its clarity: the criterion value has a direct impact on the final decision. This clarity can make this approach easier to use. A second advantage of the Basilico and Amigoni approach is that it allows one to take into account the redundancy of the criteria.

To sum up, both approaches can be relevant depending on the set of criteria used to make the decision. If some criteria require considering the fact that the impact of their difference value is non-linear, our approach is more relevant. If it is not the case or if many criteria are redundant, then the Basilico and Amigoni approach is more relevant.

\section{EXPERIMENTAL EVALUATION}

\section{A. Experimental context}

1) General context: In order to validate our approach, we propose to compare it with several known exploration strategies. In this context, we propose to compare the travelled distance necessary for each strategy to cover $90 \%$ of the total free area of several environments. The choice of this percentage (which is similar to the percentage used in [3]) reflects the fact that the last explored $10 \%$ is usually composed of corners and minor features of the environment that does not contribute significantly in evaluating strategies.

The experiments were carried out with the GAMA simulation platform [19]. This platform provides a complete modelling and simulation development environment for building spatially explicit multi-agent simulations. In particular, it integrates many built-in algorithms concerning the multicriteria decision making.

2) Robot characteristics: For the purpose of our experiments, we assume that the robot used is holonomous and is equipped with a laser range finder sensor. For the sake of

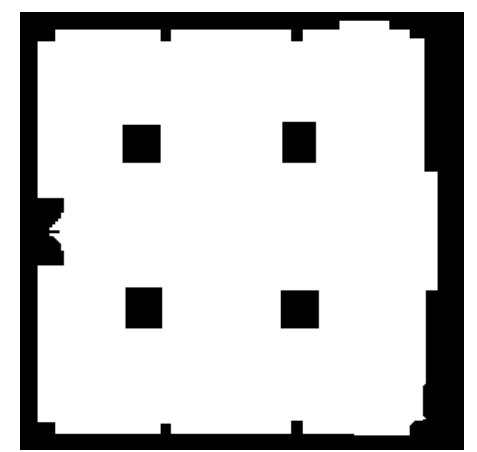

Fig. 4. Open environment used for the experiments

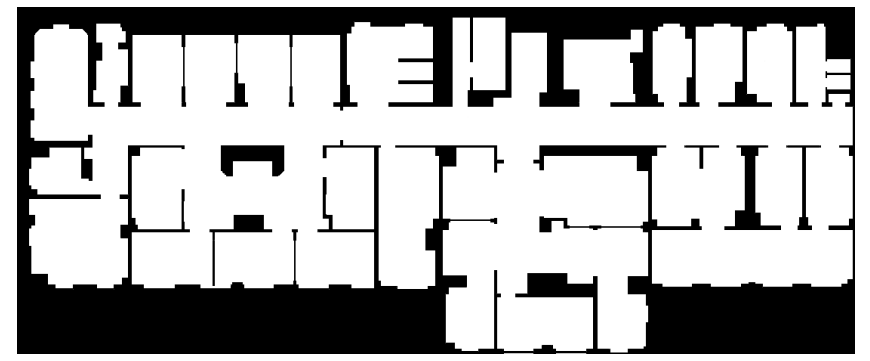

Fig. 5. Clustered environment used for the experiments

simplicity, we also assume that the robot location is known from the beginning to the end of the mission. The sensor acquire $360^{\circ}$ range date with a resolution of $0.5^{\circ}$ and a range of $5 \mathrm{~m}$. The speed of the robot is $0.5 \mathrm{~m} . \mathrm{s}^{-1}$.

3) Tested environments: In order to evaluate the different exploration strategies, we choose to use two different environments already used by [3]. They represent two usual exploration scenarios:

1) Open environment (Figure 4). The total area is approximatively $10000 \mathrm{~m}^{2}$

2) Clustered environment (Figure 5). The total area is approximatively $15000 \mathrm{~m}^{2}$

\section{B. Tested strategies}

Here is the list of the tested strategies:

1) DistMin: selection of the candidate that minimizes $L(p)$.

2) GBL: strategy proposed by Gonzales-Banos and Latombe [2]. Tested with lambda $=0.2$ (the same value reported in the original paper [2]).

3) MCDM_BA: strategy proposed by Basilico and Amigoni [4]. Tested with the three criteria defined in Section III-C and the weights found in the paper [4]:

\begin{tabular}{|l|l|}
\hline Criteria & Weigth \\
\hline L & 0.3 \\
\hline A & 0.5 \\
\hline P & 0.2 \\
\hline A, L & 0.95 \\
\hline A, P & 0.7 \\
\hline L, P & 0.4 \\
\hline
\end{tabular}




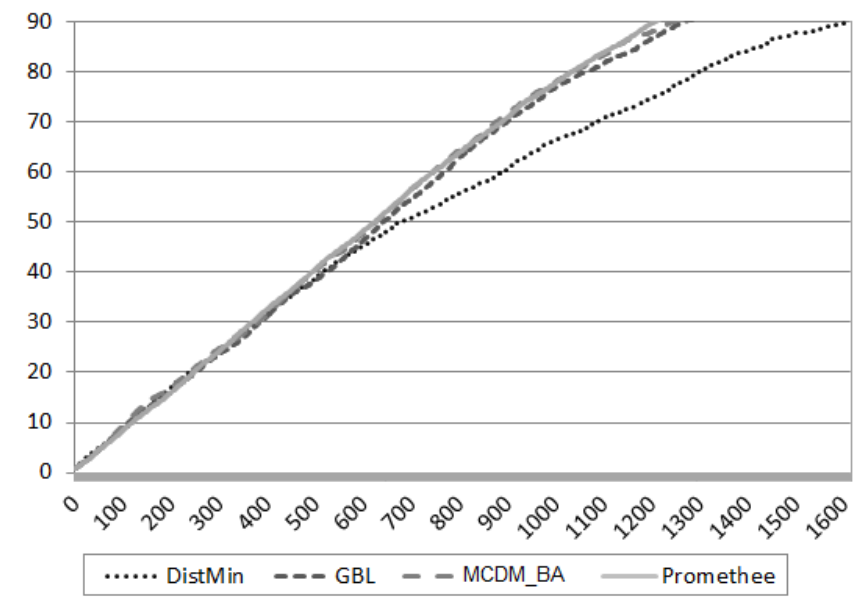

Fig. 6. Results for the open environment. X-axis: travelled distance; Y-axis: percentage of covered area

4) Promethee: Tested with the three criteria defined in Section [II-C] (with the same value for the thresholds). Tested with the following weigths:

\begin{tabular}{|l|l|}
\hline Criterion & Weigth \\
\hline L & 0.6 \\
\hline $\mathrm{A}$ & 0.3 \\
\hline $\mathrm{P}$ & 0.1 \\
\hline
\end{tabular}

The weights were assigned manually. We chose to assign more importance to $L$ than to $A$ and $P$ in order to limit back-and-forth movements. Concerning the two other criteria, we chose to assign more importance to $A$ than to $P$ in order to push the robot to discover new areas, even if risking a loss of communication.

\section{Results}

1) Open environment: The results obtained with the different exploration strategies are presented Figure 6. It corresponds to the average results obtained after 10 simulations.

We can observe that the best results were obtained with the PROMETHEE strategies (1208m travelled to cover $90 \%$ of the total free area). MCDM_BA and GBL strategies obtained close results, but not quite as good (respectively, $1244 \mathrm{~m}$ and $1270 \mathrm{~m}$ travelled to cover $90 \%$ of the total free area). The DistMin strategy obtained bad results in comparison to the other strategies (more than $1600 \mathrm{~m}$ travelled to cover $90 \%$ of the total free area).

Figure 7 shows the path followed by the robot for the open environment while using the PROMETHEE exploration strategy.

2) Clustered environment: The results obtained with the different exploration strategies are presented Figure 8 . It corresponds to the average results obtained after 10 simulations.

We can observe that the best results were obtained with the PROMETHEE and the GBL strategies (respectively, $2961 \mathrm{~m}$ and $3032 \mathrm{~m}$ travelled to cover $90 \%$ of the total free area), the worse results with the DistMin strategy (more than $3200 \mathrm{~m}$

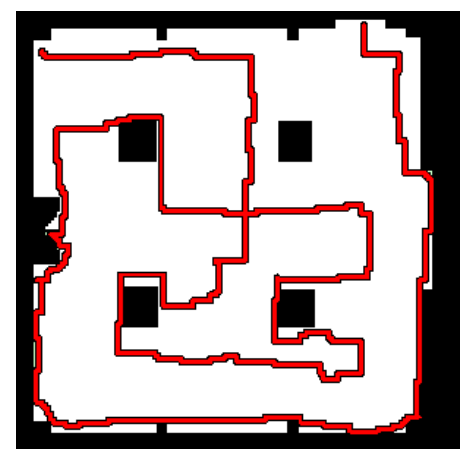

Fig. 7. In red: the path followed with the PROMETHEE strategy for the open environment

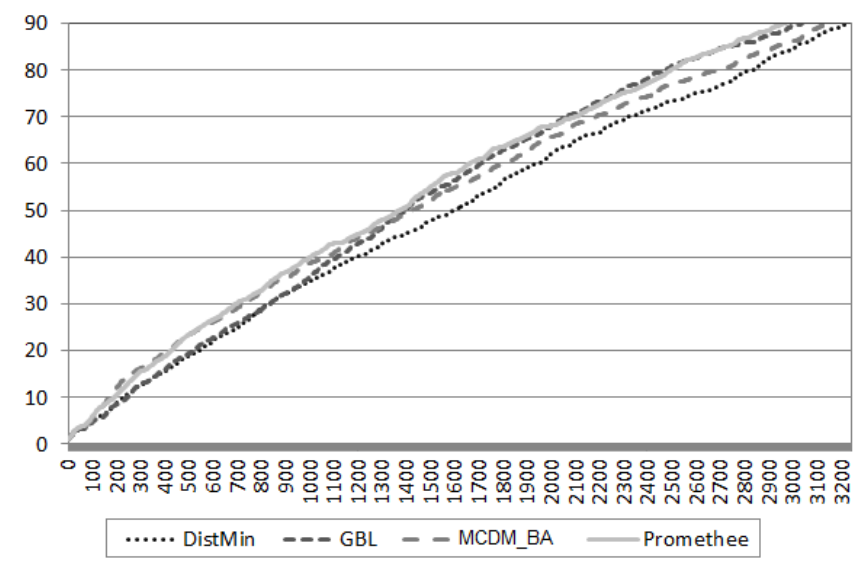

Fig. 8. Results for the clustered environment. $\mathrm{X}$-axis: travelled distance; Y-axis: percentage of covered area

travelled to cover $90 \%$ of the total free area). The MCDM_BA strategy obtained results close to the DistMin strategy (3142m travelled to cover $90 \%$ of the total free area).

Figure 9 shows the path followed by the robot for the clustered environment while using the PROMETHEE exploration strategy

\section{Conclusion about the experiments}

We can observe that the best results were obtained with the PROMETHEE strategy. The GBL and MCDM_BA strategies allowed to obtain good result. The results obtained with the DistMin strategy were the worst for both environments.

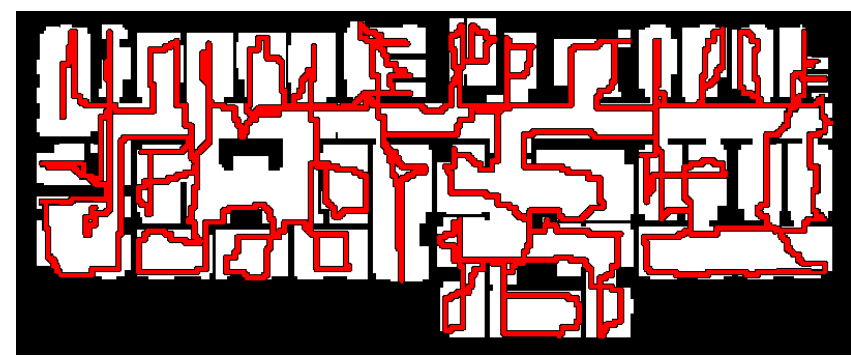

Fig. 9. In red: the path followed with the PROMETHEE strategy for the clustered environment 
To conclude, these experiments show that our approach is very effective and permits us to get very good exploration results. Moreover, one of the other advantages of our approach (and of the MCDM_BA strategy) is that it is based on a well-established multi-criteria decision making framework that allows easy addition of new criteria.

\section{CONClusion}

In this paper, we proposed an new approach to combine several criteria to select the best candidate location for NBV exploration algorithms thanks to the PROMETHEE II multicriteria decision making method. Experimental result show that our approach can allow to obtain better results than classic NBV approaches such as GBL [2] and close to other MCMD work [4].

An important issue when using a multi-criteria decision making method concerns their parametrization. In fact, the quality of the decision making is directly linked to the relevance of the values chosen for the parameters. It is thus important to choose relevant parameter values. Several works propose approaches to elicit parameters values. In our context, an approach based on Machine Learning such as the one proposed by [20] seems to be very suitable.

A perspective would be to test other multi-criteria decision making methods. Indeed, in this paper, we propose to use the PROMETHEE II method, which is well-suited for our problem. However, other methods such as the ones based on the Dempster-Shafer theory [21] (e.g. the one proposed in [22]) could also be used.

Another perspective of this work would be to define new criteria. In particular, criteria specially dedicated to rescue mission. For example, it could be interesting to take into account information such as the type of buildings in the area (e.g. during the night, most of the disaster victims are located in residential areas).

\section{ACKNOWLEDGMENT}

This work has been done in the context of the AROUND (Autonomous Robots for Observation of Urban Networks after Disasters)[23] project. It has been so far supported by the ICT-Asia progran 1 IRD (Institut de la Recherche pour le Développement, France), AUF (Agence Universitaire de la Francophonie) and École des Mines de Douai (France).

\section{REFERENCES}

[1] B. Yamauchi, "Frontier-based exploration using multiple robots," in Proceedings of the Second International Conference on Autonomous Agents, pp. 47-53, 1998.

[2] H. H. Gonzáles-Baños and J.-C. Latombe, "Navigation strategies for exploring indoor environments," International Journal of Robotics Research, vol. 21, no. 10-11, pp. 829-848, 2002.

[3] N. Basilico and F. Amigoni, "Exploration strategies based on multicriteria decision making for an autonomous mobile robot," in Proceedings of the European Conference on Mobile Robots (ECMR), pp. 259264, 2009 .

${ }^{1}$ French Ministry of Foreign Affairs/INRIA/CNRS/IRD
[4] N. Basilico and F. Amigoni, "Exploration strategies based on multicriteria decision making for search and rescue autonomous robots," in Proc. of 10th Int. Conf. on Autonomous Agents and Multiagent Systems (AAMAS 2011), pp. 99-106, 2011.

[5] C. Stachniss and W. Burgard, "Exploring unknown environments with mobile robots using coverage maps," in Proceedings of the International Conference on Artificial Intelligence (IJCAI), 2003.

[6] F. Amigoni, V. Caglioti, and U. Galtarossa, "A mobile robot mapping system with an information-based exploration strategy," in Proceedings of the International Conference on Informatics in Control, Automation and Robotics (ICINCO 2004), pp. 71-78, 2004.

[7] W. Burgard, M. Moors, C. Stachniss, and F. Schneider, "Coordinated multi-robot exploration," IEEE Transactions on Robotics, vol. 21, no. 3, pp. 376-386, 2005.

[8] B. Roy, Méthodologie multicritère d'aide à la décision. Paris : Economica, 1985

[9] B. Roy, "The outranking approach and the foundations of electre methods," Theory and Decision, vol. 31, pp. 141-160, 1991.

[10] G. Siskos, J. Wäscher and H. Winkels, "A bibliography of outranking approaches," tech. rep., Cahiers du Lamsade, Paris Dauphine, 1983.

[11] J. P. Ignizio, "A review of goal programming: a tool for multiobjective analysis," Journal of the Operational Research, vol. 29, no. 11, pp. 1109-1119, 1978

[12] E. Jacquet-Lagreze and J. Siskos, "Assessing a set of additive utility functions for multicriteria decision making, the uta method," Eur. J. Oper. Res., vol. 10(2), pp. 151-164, 1982.

[13] M. Grabisch and C. Labreuche, "A decade of application of the choquet and sugeno integrals in multi-criteria decision aid," 4OR: A Quarterly Journal of Operations Research, vol. 6, pp. 1-44, 2008. 10.1007/s10288007-0064-2.

[14] R. Benayoun, O. Laritchev, J. de Mongolfier, and J. Tegny, "Linear programming with multiple objective functions: STEP method (STEM)," Mathematical Programming, vol. 1, no. 1, pp. 366-375, 1971.

[15] A. Geoffrion, J. Dyer, and A. Feinberg, "An interactive approach for multicriterion optimisation with an application to the operation of an academic department," Management Science, vol. 19, no. 4, pp. 357368, 1972.

[16] T. Al-Shemmeria, B. Al-Klouba, and A. Pearman, "Model choice in multicriteria decision aid," European Journal of Operational Research, vol. 97 , no. 3, pp. 550-560, 1997.

[17] M. Behzadian, R. Kazemzadeh, A. Albadvi, and M. Aghdasi, "PROMETHEE: A comprehensive literature review on methodologies and applications," European Journal of Operational Research, vol. 200, no. 1, pp. 198-215, 2009.

[18] J.-P. Brans and B. Mareschal, "PROMETHEE methods," in Multiple Criteria Decision Analysis: State of the Art Surveys (J. Figueira, S. Greco, and M. Ehrgott, eds.), vol. 78 of International Series in Operations Research \& Management Science, Springer, 2005.

[19] P. Taillandier, D.-A. Vo, E. Amouroux, and A. Drogoul, "GAMA: a simulation platform that integrates geographical information data, agentbased modeling and multi-scale control," in Proceedings of the 13th International Conference on Principles and Practices in Multi-Agent Systems (PRIMA), 2010.

[20] K. Jabeur and A. Guitouni, "Automated learning multi-criteria classifiers for flir ship imagery classification," in Proceedings of the 10th International Conference on Information, 2007.

[21] G. Shafer, A mathematical theory of evidence. Princeton University Press, 1976.

[22] P. Taillandier, C. Duchêne, and A. Drogoul, "Using belief theory to diagnose control knowledge quality: Application to cartographic generalisation," in Proceedings of the International Conference on Computing and Communication Technologies (RIVF'09), 2009.

[23] A. Boucher, R. Canal, T.-Q. Chu, A. Drogoul, B. Gaudou, V. T. Le, V. Moraru, N. V. Nguyen, Q. A. N. Vu, P. Taillandier, F. Sempé, and S. Stinckwich, "The AROUND project: Adapting robotic disaster response to developing countries," in Proceedings of 2009 IEEE International Workshop on Safety, Security, and Rescue Robotics, IEEE Computer Society, 2009. 